\title{
Response to Commentaries on Sudden Gains and Sudden Losses in the Clients of a "Supershrink": 10 Case Studies
}

\section{Calling for More Case Studies of Exceptional and Efficient Psychotherapists}

\author{
BRIAN P. HANSEN, ${ }^{\mathrm{a}}$ MICHAEL J. LAMBERT, ${ }^{\mathrm{a}, \mathrm{c}}$ \& ERIGONI N. VLASS ${ }^{\mathrm{b}}$ \\ a Department of Psychology, Brigham Young University, Provo, Utah \\ ${ }^{\mathrm{b}}$ Private Practice, Sydney, Australia \\ ${ }^{\mathrm{C}}$ Correspondence regarding this article should be addressed to Michael J. Lambert, 272 Taylor Building, Provo, UT. \\ Email: Michael_lambert@byu.edu \\ Acknowledgement. We wish the thank Daniel B. Fishman, Editor of the PCSP journal, for his patient guidance and \\ wisdom as we worked through various versions of our manuscripts and phases of publication. He is truly committed \\ to editing in the best sense of this word-more of a partner than harsh critic.
}

\begin{abstract}
We respond to issues raised in the commentaries on early response and the exceptional psychotherapist, noting that our findings are consistent with those suggested by Laska and Federman (2015) as well as by Pereira and Barkham (2015). We call for prospective study of exceptional therapists that employ the variables and constructs noted in the commentaries in addition to retrospective recall of change events.

Key words: sudden gains; rapid response; rapid responders; therapy outcome; off-track; sudden loss; deteriorators; therapist factors; mixed methods; supershrink; outcome questionnaire (OQ-45); measuring change; case studies; clinical case studies

We wish to thank the commentators (Laska \& Federman, 2015; and Pereira \& Barkham, 2015) for their thoughtful and constructive review of our case study of an exceptional therapist (Hansen, Lambert, \& Vlass, 2015). We found the reviews to be stimulating and thought provoking, both with regards to the many factors that can influence patient outcome as well as two important contemporary issues in psychotherapy outcome: early dramatic response and the effects of the individual psychotherapist on outcome. We have little to add to their comments, which raise methodological issues, reasoned opinion and speculation about our case study. We offer some important clarifications.

One major difference in focus between the Laska and Federman (2015) and Pereira and Barkham reviews is the extent to which they focus on different aspects of our paper-with Laska and Federman emphasizing issues related to early gains, and Pereira and Barkham emphasizing the characteristics of the effective therapist. We organize our response to these commentaries around these two topics.
\end{abstract}




\section{EARLY GAINS}

We are in agreement with Laska and Federman (2015) that early gains in psychotherapy are an important phenomenon. Their review of literature in this area is consistent with our own understanding based on reading much of the same body of research. Early gains occur in a surprisingly high proportion of clients; early gains are positively related to final outcome and to psychological health at follow-up; and early gains occur across different kinds of psychopathology and a variety of psychological treatments (Haas, Hill, Lambert, \& Morrell, 2002).

Early gains may be related to the construct of "corrective emotional experience" where there is broad consensus across therapists and therapy researchers of different theoretical orientations and generations that change during psychotherapy is often not just small incremental steps towards improvement, but not uncommonly much more dramatic change at particular times. Although it has been more than 60 years since Alexander and French (1946) proposed corrective emotional experiences (or simply "corrective experiences" [CEs]) as the key change process in psychoanalysis and psychotherapy, research has generally ignored CEs as a central part of the change process (Castonguay \& Hill, 2013). CEs happen anytime during the course of psychotherapy, but it was such changes that drew our (BPH \& MJL) attention to the work of Eri Vlass. Not only did many of her patients show unusually dramatic change between sessions, but also these appeared very early on in the course of treatment. While such changes may be a manifestation of corrective emotional experiences, CEs imply a process of change that is dramatic, while early response simply reflects an unusually large improvement very early in the therapy process.

Such early gains are particularly interesting because they cannot be solely due to specific treatment techniques since they occur generally across psychotherapies and prior to instigation of many techniques that are presumably necessary for successful treatment, including compassionate mind therapy as practiced by Eri. Early dramatic gains deserve the attention of both clinicians and researchers. Since Eri seems to produce an inordinate number of such responders, her work with clients may continue to be worthy of study and, particularly, in prospective research.

In addition to early gains following the first session, Laska and Federman also raise the question of those gains even preceding first session contact with the therapist. This is a rarely studied and reported in the research literature. Unfortunately, we did not study this possibility by giving the OQ-45 week(s) prior to the first treatment session. If responding before the first session is common, it raises the likelihood that improvement attributed directly to psychotherapy or the psychotherapist (and in this case Eri) is due to the relief that comes from making the decision to enter treatment and taking action by scheduling an appointment. We can hypothesize that the rate at which pre-therapy gains occur ought to be constant across therapists unless the person(s) who makes the referral provides such a strong endorsement that clients expectations for a positive outcome are enhanced beyond the ordinary. For many clients, when they make the decision to enter treatment. change may already be taking place-something that might be thought of as moving from a state of distress and disorganization to implementing 
actions towards self-reorganization (Heinzel \& Schiepek, 2014). Some therapists with particularly strong reputations (such as Eri) may have greater impact on patients even prior to the first contact; and this should not be attributed to the therapeutic actions taken with a particular client, but could instead come from outcomes in prior patients who then make enthusiastic referrals or give highly positive feedback to the referring physician. Certainly, pre-therapy gains need to be considered and perhaps they impact on the estimate of therapist effects. As far as we know this has never been studied.

As Laska and Federman (2015) suggest a wise place to search for causal variables that could account for early gains (and losses) is within the characteristics that clients bring with them to the psychotherapy enterprise. They argue, based on accumulated literature, that we can expect early gain rates to vary by diagnosis as well as indicators of chronicity and co-morbidity. By comparing Eri's case load to that of a university counseling center used in our earlier search for "supershrinks" (Okiishi, Lambert, Nielsen, \& Ogles, 2003), it is obvious that Eri is treating a far different (more disturbed) population based on initial OQ-45 scores.

However, in the Okiishi et al. study we compared therapists on the basis of client outcome at the time of termination but did not calculate the proportion of clients who made early gains or early losses on a therapist-by-therapist basis. Thus we do not know the range or central tendency of early gains and losses from that study. We do know that clients were, on average, far less disturbed (as measured by the OQ-45) at intake than were Eri's patients and that it is more difficult to measure dramatic changes in individuals who are functioning in the normal range.

Laska and Federman present a table highlighting key differences between Eri's Red and Blue populations, and it contributes important insight into possible client variables that could account for success or deterioration in therapy. Laska and Federman imply that the rapid response or sudden loss experienced by Eri's clients is better attributed to pre-existing client variables, and less to the effectiveness of Eri's therapeutic interventions. While we understand their rationale and their use of the clinical material as presented, we believe that there is reason to not take their interpretation of the clinical material at face value. It should be noted that much of their data for distinguishing between the two populations is derived from Eri's self descriptions of her own clients, which were an amalgamation of retroactive treatment-note review and posthoc hypotheses regarding change mechanisms. Given that Eri cannot extract herself from her own experiences with her clients to view her clients more objectively, her recollection of clients' baseline functioning (including variables such as latent resiliency, situational vs. chronic stress, and even diagnostic accuracy) may have been affected by her memory of the client's progress in therapy. In other words, Eri might be prone to an observer bias effect as a result of her subjective experience with a patient. It is a well-documented phenomenon that therapists tend to like their patients more when they are making progress in therapy. Thus, Eri's retroactive account of pre-treatment functioning may be distorted through the lens of her recollection and fondness for the client. Her analysis of the patient's resiliency and personal resourcefulness may not accurately reflect genuine differences between the client populations. The bottom line is that Laska and Federman raise an important hypothesis that needs to be assessed in future studies by measuring pre-treatment variables beyond symptomatic distress. 
Pereira and Barkham (2015; p. 217), in their discussion of sudden gains and sudden losses, seem to fail to differentiate between (a) the concept of defining a reliable change statistically (worsening by 14 points or more on the OQ- 45 and 5 or more points on the CORE), and (b) procedures used to identify cases that are identified as Not-On-Track (red cases) and who show sudden losses. Although possibly just a technical point, the difference between reliable change cut-off scores and cut-off scores for identifying "red" and "blue" cases are based on algorithms, not reliable change. We use Jacobson and Truax's (1991) formula for the Reliable Change Index to define termination status (patient outcome). But the score used to identify a blue case or a red case is not the RCI, which is an absolute score, but rather the algorithms we use reflect that a particular patient, with a particular intake score, at a particular session of therapy, has responded to treatment that is characteristic of the best $10 \%$ of responders to treatment. Thus, if a patient who begins treatment reporting a great deal of disturbance and at the $20^{\text {th }}$ session of treatment has not improved or worsened they could be identified as a red case because $90 \%$ of patients show some improvement by that session of care.

In addition, the OQ-Analyst contains an additional method of identifying red cases based on clinical consensus, rather than infrequent poor treatment response, so called "rational algorithm” (see Lambert, Whipple, Bishop, 2002). This particular method for identifying cases at risk for treatment failure is not subject to the statistical problems raised by Pereira and Barkham. For example, a patient who enters treatment reporting nearly unbearable levels of pain (e.g., an OQ score of 120), who reports just slight improvement after 20 sessions of treatment would be considered at risk of treatment failure by the rational algorithms (i.e., most clinicians would expect some positive response after that much therapy and be very concerned about upcoming suicide or other "impulsive" behavior). We have not developed a rational method for identifying sudden gains because positive treatment response has not been a concern in our research endeavors.

\section{THE EFFECTIVE THERAPIST}

After suggesting five things Eri did, and effective therapists in general do, Pereira and Barkham (2015, p. 219) raise some interesting questions about other things that they have found to distinguish effective therapists. First, they want to know more about the nature of Eri's experiences as a therapist and, in particular, the extent to which she participates in activities that inform her practice such as peer supervision and related professional activities. Second, they would like to have a detailed explanation of her "state of being" during her sessions. They hope to better understand how consistent her practice is with their findings - that effective therapists practice in a mindful state and are open learners.

Here is Eri's response to their questions:

I am very vigilant with self care. I maintain a healthy lifestyle exercising everyday, eating healthy foods and getting adequate sleep. I practice mindfulness meditation. During treatment sessions I am very focused on the person I am working with and no matter how difficult the presenting problem or the underlying problem for that matter, I know that I will be able to respond appropriately. I have had extensive clinical experience over the past twenty years working in areas such a post natal depression, sexual health, child and adolescent anxiety, 
trauma, drug and alcohol, eating disorders, bereavement and loss, sleep disorders, anxiety and depression, family therapy and parenting. As a member of the Australian Psychological Society we are required to complete 30 hours of professional development a year, which means that we attend conferences workshops and engage in peer supervision.

In our (BPH \& MJL) reflection about Eri: She appears to be constantly searching for ways of improving her therapy skills, including measuring and monitoring her patients' treatment response. She is consistently enthusiastic about being a psychotherapist and optimistic about her patients' abilities to improve the quality of their lives.

An important issue raised by Laska and Federman's commentary concerns the generalizability of the source article (Hansen, Lambert, \& Vlass, 2015). They note in their final remarks that Eri's dramatic response rate in therapy is 2.8 times more powerful than that which is typically seen amongst sudden gain patients in other settings, and thereby conclude: "This suggests that not only do the Blues in Eri's sample come from a different population than the Reds, but that they come from a different population from those who present to a University Counseling Center in the U.S.” We concede that a more complete comparison of Eri's entire caseload to that of other outpatient settings would be important to determine whether their hypothesis is correct. However, we must point out that a difference in relative outcomes between different populations does not necessarily indicate a difference in population demographics. Indeed, Eri's Blue clients actually entered therapy with a higher-than-average symptom severity than has been observed in most outpatient populations. Her exceptional outcomes may be due in part (as is postulated by Laska and Federman) to differences in client demographics within Eri's caseload; in other words, her selective sample may not be representative of the population at large. However, her outcomes may also be due to unique therapist characteristics that make her an exceptionally effective therapist. Further studies should examine the effects of base-line client variables to determine whether Blue and Red cases represent two different populations.

Pereira and Barkham proposed an effective method for assessing clients pre-treatment status with measures of a client's latent resiliency and mindfulness, using scales such as the Connor-Davidson Resilience Scale and measures of Mindfulness. Future studies might consider taking this suggestion into account, and more thoroughly examine pre-treatment client variables that may predict success in therapy. However, based on our experience in working with therapists with exceptional results like Eri, we expect that further investigations of such therapists will show that they will be characterized by unusually high rates of blue cases and low rates of red cases compared to their peers after pre-treatment differences are controlled for, e.g. by random assignment.

When comparing Eri's therapeutic response rate to that of other therapists', it is important to differentiate between the two overlapping, yet unique constructs of "rapid response" vs. "sudden gains." It should be noted that the algorithms, analysis, and description tools available in the OQ-45 allow for an examination of both constructs, albeit through different means. Whether a patient is designated as a "Red" or "Blue" case does not refer to rapid-response necessarily; it merely indicates how this particular patient is progressing in treatment, at a particular session of therapy, when compared to a normative sample of other patients who entered therapy with the 
same level of distress. So the presence of a "Blue or Red" status is a comparative distinction, evaluating the client's current progress at a particular point in therapy compared to their cohort. This construct differs from that of "rapid response," which implies dramatic improvement in a short period of time. The algorithms of the OQ-45 do not explicitly indicate whether response is "rapid" or "slow." This can only be obtained through a post-hoc analysis of when (i.e. at which session of therapy) significant improvement on the OQ-45 occurred (determined by the researcher or clinician's definition of "rapid” response). Given that several different criteria have been proposed for "rapid" vs. "sudden” response, it necessitates the researcher or clinician to clarify terms before undertaking an analysis of the patients' change trajectory.

We hope that the reviewers and we have sparked the imagination and curiosity of psychotherapists and psychotherapy researchers and that both are able to build upon this work and take us to a new level in our understanding and appreciation of how clients change.

\section{REFERENCES}

Alexander, F. and French, T. M. (1946). Psychoanalytic therapy. New York: Ronald Press. Castonguay, L. G. \& Hill C. E. (Eds.) (2013). Transformation in psychotherapy: Corrective experiences across cognitive behavioral, humanistic, and psychodynamic approaches. Washington DC: American Psychological Association.

Haas, E., Hill R., Lambert, M. J., \& Morrell, B. (2002). Do early responders to psychotherapy maintain treatment gains? Journal of Clinical Psychology, 58, 1157-1172.

Hansen, B. P., Lambert, M. J., \& Vlass, E. N. (2015). Sudden gains and losses in the clients of a "supershrink": 10 case studies. Pragmatic Case Studies in Psychotherapy, 11(3), Article 1, 154-201. Available: http://pcsp.libraries.rutgers.edu

Heinzel, S. \& Schiepek, G. (2014). Dynamic patterns in psychotherapy-discontinuous changes and critical instabilities during the treatment of obsessive-compulsive disorder. Nonlinear Dynamics, Psychology, and Life Sciences, 18, 155-176.

Jacobson, N.S., \& Truax, P. (1991). Clinical significance: A statistical approach to defining meaningful change in psychotherapy research. Journal of Consulting and Clinical Psychologv, 59, 12-19.

Lambert, M. J., Whipple, J. L., Bishop, M. J., Vermeersch, D. A., Gray, G. V., \& Finch, A. E. (2002). Comparison of empirically derived and rationally derived methods for identifying clients at risk for treatment failure. Clinical Psychology and Psychotherapy, 9, 149 -164.

Laska, K. M. \& Federman, E. J. (2015). Rapid recovery with an effective therapist: A comment on Hansen, Lambert, and Vlass. Journal of Pragmatic Case Studies in Psychotherapy, 11(3), Article 2, 202-215. Available: http://pcsp.libraries.rutgers.edu

Okiishi, J., Lambert, M.J., Nielsen, S.L., \& Ogles, B.M. (2003). Waiting for supershrink: An empirical analysis of therapist effects. Clinical Psychology \& Psychotherapy, 10, 361373.

Pereira, J. \& Barkham, M. (2015). An exceptional, efficient, and resilient therapist: A case study in practice-based evidence. Journal of Pragmatic Case Studies in Psychotherapy, 11(3), Article 3, 216-223. Available: http://pcsp.libraries.rutgers.edu 